\title{
Optimization of curing cycle in carbon fiber-reinforced laminates: Void distribution and mechanical properties
}

\author{
S. Hernández , F. Sket “, C. González ־,-, J. LLorca
}

\begin{abstract}
A B S T R A C T
A strategy is presented to optimize out-of-autoclave processing of quasi-isotropic carbon fiber-reinforced laminates. Square panels of $4.6 \mathrm{~mm}$ nominal thickness with very low porosity $(\leqslant 0.2 \%)$ were manufactured by compression molding at low pressure $(0.2 \mathrm{MPa})$ by careful design of the temperature cycle to maximize the processing window. The mechanisms of void migration during processing were ascertained by means of X-ray microtomography and the effect of ply clustering on porosity and on void shape was explained. Finally, the effect of porosity and ply clustering on the compressive strength before and after impact was studied.
\end{abstract}

\section{Introduction}

High performance carbon fiber-reinforced composites are manufactured by the consolidation at high temperature of laminates made up of prepreg plies with different fiber orientation. Consolidation is normally carried out in an autoclave because the high hydrostatic pressure ( $\geqslant 0.7 \mathrm{MPa}$ ) ensures full compaction of the laminate, leading to a maximum fiber volume fraction and negligible porosity and, thus, to optimum mechanical properties. Autoclave processing has, however, major drawbacks from the industrial viewpoint (long processing time, limited production rate and component size, high capital investment) and there is an enormous interest in optimizing out-of-autoclave processing routes so high performance composites can be processed at higher rates and lower cost [1-5].

Processing of thermoset laminates is carried out by the application of a cure cycle which is defined by the temperature and pressure profile. Pressure and temperature are the driving forces to bleed the laminate, consolidate individual plies and reduce the void content. The temperature initially reduces the viscosity of the resin, favoring the impregnation of the fibers, but it also triggers the crosslinking reactions which ultimately lead to the gelification of the resin. The external pressure drives the excess resin and air bubbles out of the laminate. The mechanics of prepreg compaction in autoclave was pioneered by Springer and co-workers [6,7] starting from the consolidation theory developed for soil mechanics [8]. These authors described the resin flow through the composite following Darcy's flow theory in a porous medium, and determined the laminate compaction sequence. The external pressure was first supported by the resin and, as bleeding progressed through the surfaces, pressure was transferred to the fiber bed. This process continued until the composite reached the maximum compaction of the reinforcement and all the extra resin was expelled. Air bubbles are always present in the raw prepreg due to deficient fiber impregnation and others arise on the lamina surface during the preparation of the laminate kit, leading to an initial void population that is subjected to the curing cycle. The stability of voids as a function of the temperature and pressure has been extensively studied by Kardos et al. [9], who considered the effects of the resin viscosity and of the resin-void surface tension. They developed a model for void growth which was successfully applied to predicting the occurrence of voids in thermoset composite materials manufactured by liquid molding [10], autoclave or vacuum bagging [3]. Although these models provide the essentials of the mechanics of void growth in a polymer blend, they are restricted to small spherical voids surrounded by a viscous resin. Their validity is not proven for long cylindrical voids in a viscous matrix surrounded by aligned fibers, the standard morphology observed in out-of-autoclave composites. In addition, under outof-autoclave processing conditions (compression molding, 
consolidation under atmospheric pressure), the maximum pressure is limited and the manufacturing of high performance carbon-fiber composites requires the design of sophisticated temperature cycles, based in a detailed analysis of the resin curing kinetics, to obtain fully consolidated, void-free laminates.

An example of this strategy was presented in a previous investigation [4]. Starting from a detailed characterization of the rheology and curing kinetics of the raw prepregs, several cure cycles were designed and unidirectional panels were consolidated using these cycles. It was found that longer processing windows (more time before gelation) led to laminates with lower fraction of voids for simple temperature cycles (involving only ramps and holds) while the final void volume fraction also depended on the actual evolution of the viscosity throughout the cycle in the case of complex cycles, involving flash temperatures followed by holds at lower temperatures. Finally, the effect of curing cycle and void volume fraction on the interlaminar shear strength was studied. In this investigation, the previous work was expanded to deal with quasi-isotropic composite panels with dispersed and clustered ply lay-ups, similar to those used in industrial applications. They were consolidated using two cure cycles optimized for out-of-autoclave processing as well as the standard cycle recommended for autoclave. The effect of curing cycle and of laminate lay-up on the volume fraction, shape and spatial distribution of the voids was determined by means of X-ray computed microtomography (XCT). Moreover, this information was used to infer the mechanisms of void migration and coalescence in quasi-isotropic laminates during consolidation. Finally, the compressive strength of the panels before and after impact was measured to assess the influence of the cure cycle on the mechanical behavior.

\section{Materials and processing}

Unidirectional carbon/epoxy AS4/8552 prepreg sheets were purchased from Hexcel. The 8552 epoxy system was modified by the supplier by incorporating of thermoplastic particles to enhance fracture toughness and impact performance [11]. The nominal prepreg areal weight was $194 \mathrm{~g} / \mathrm{m}^{2}$. Square $\left(340 \times 340 \mathrm{~mm}^{2}\right)$ panels with $4.4 \mathrm{~mm}$ nominal thickness were manufactured by compression molding (Fontijne Grotnes LabPro400). The prepreg plies were stacked using two different quasi-isotropic lay-ups, $\left[45_{3} / 0_{3} /-45_{3} / 90_{3}\right]_{s}$ and $[45 / 0 /-45 / 90]_{3 s}$, to analyze the effect of ply clustering on the consolidation process and on the mechanical properties. Laminate kits were placed between polytetrafluoroethylene sheets for adequate release after the consolidation. No previous vacuum debulking of the laminate prepreg kit was performed and a constant pressure of $0.2 \mathrm{MPa}$ was applied immediately after placing the kit between the press plates. Three different temperature cycles, detailed below, were applied to cure the multiaxial composites.

\section{Experimental techniques}

\subsection{Rheology and thermal analysis}

Rheological measurements under dynamic mode were performed using a parallel plate rheometer (AR200EX, TA Instruments) with disposable plates. A prepreg raw sample of $25 \mathrm{~mm}$ in diameter and 4 plies in thickness was placed between the plates of the rheometer and subjected to an oscillatory shear strain of $0.05 \%$ and $1 \mathrm{~Hz}$ frequency while imposing a specified temperature cycle. The dynamic viscoelastic response of the composite prepreg in terms of the storage and loss moduli, $G^{\prime}$ and $G^{\prime \prime}$, respectively, is modified by the cross-linking reactions and the evolution of the complex viscosity $\eta^{*}$ (modulus of real and imaginary parts) can be determined as

$\eta^{*}=\frac{\left|G^{\prime}+i G^{\prime \prime}\right|}{\omega}$

where $\omega$ is the frequency of the oscillatory strain. The presence of the fibers in the prepreg increased storage component of the shear modulus and it was not possible to determine the gel point using the standard criterion for neat resins, i.e. $G^{\prime}=G^{\prime \prime}$, because these curves never cross each other. Thus, the gel point was assumed to be reached when $\tan \delta=G^{\prime \prime} / G^{\prime}$ was independent of the applied frequency, according to the criterion developed by Winter and Chambon [12]. Additionally, the dynamic viscoelastic response of the neat 8552 epoxy resin was compared with that of the prepreg in order to evaluate the effect of the fiber reinforcement on the rheological properties. Both viscosity profiles presented similar trends and the only difference was the higher value of the storage component in the prepreg due to the fibers.

Differential scanning calorimetry (DSC) was used to measure the degree of curing, $\alpha$, and the glass transition temperature, $T_{g}$, of the composite panels. The calorimetric experiments were carried out using a modulated differential scanning calorimeter (MDSC Q200, TA Instruments) using raw prepreg samples $(10 \mathrm{mg})$ subjected to the corresponding temperature cycle. After cooling down to room temperature, the specimens were reheated at $5^{\circ} \mathrm{C} / \mathrm{min}$ in a modulated mode (amplitude $\pm 2 \mathrm{~s}$ and period $100 \mathrm{~s}$ ) in order to obtain the residual reaction heat, $\Delta H_{\text {res }}$, and the glass transition temperature, $T_{\mathrm{g}}$. The final degree of curing of each sample was computed as [6]

$\alpha=1-\frac{\Delta H_{\text {res }}}{\Delta H_{\text {total }}}$

where $\Delta H_{\text {total }}$ is the total reaction heat of AS4/8552 prepreg (which was assumed to be equivalent to the dynamic reaction heat, $\Delta H_{\text {din }}$ ). The total reaction heat, $\Delta H_{\text {total }}\left(=\Delta H_{\text {din }}\right)$ of $176.20 \pm 0.48 \mathrm{~J} / \mathrm{g}$ was obtained by averaging the total enthalpy measured from room temperature up to $300^{\circ} \mathrm{C}$ at different heating rates of 5,8 and $10^{\circ} \mathrm{C} /$ min. The residual reaction heat, $\Delta H_{\text {res }}$, and the glass transition temperature, $T_{g}$, were measured by means of modulated DSC tests at $5^{\circ} \mathrm{C} / \mathrm{min}$ with $\pm 2{ }^{\circ} \mathrm{C}$ amplitude of temperature modulation and $100 \mathrm{~s}$ of period after the sample was subjected to the corresponding cure cycle.

Finally, the final volume fraction of carbon fiber reinforcement was measured using thermogravimetric experiments performed in a vertical thermobalance (model Q50, TA Instruments). The nominal fiber volume fraction of the composite panels was obtained from the densities of carbon $\left(1.79 \mathrm{~g} / \mathrm{cm}^{3}\right)$ and epoxy resin $\left(1.3 \mathrm{~g} / \mathrm{cm}^{3}\right)$ without considering the void volume fraction.

\subsection{Mechanical characterization}

Plain compression tests of the multiaxial panels were carried out according to ASTM Standard D3410. Prismatic coupons of $145 \times 20 \times 4.4 \mathrm{~mm}^{3}$ were used. They were protected with glass fiber tabs to prevent premature failure in the grip area. The load was applied to the specimen through shear by means of wedge grips. A gage length of $10 \mathrm{~mm}$ was used to prevent compressive buckling. The tests were performed at a crosshead speed of $1.5 \mathrm{~mm} / \mathrm{min}$ using an electromechanical universal testing machine (Instron 3384). The load was continuously monitored during the test with a $150 \mathrm{kN}$ load cell while the longitudinal strain along the loading axis was measured with two standard resistive strain gages attached to both lateral surfaces of the specimen.

The compressive stress was determined from the applied load and the initial cross-section while the compressive strain was obtained by averaging the data from both strain gages. 
The bending component, given by the difference in the readings of both strain gages, was always below $10 \%$. The compressive elastic modulus was obtained from the slope of stress-strain curves between 1000 and 3000 microstrains and the compressive strength from the maximum load.

Damage in the panels was introduced by means of a dropweight impact test (Instron Dynatup 8250) according to ASTM standard D7136. Rectangular specimens of $150 \times 100 \times 4.6 \mathrm{~mm}^{3}$ were machined from the quasi-isotropic AS4/8552 composite panels. The specimen was simply supported by the fixture and held at the corners with clamping tweezers, leading to a free impact area of $125 \times 75 \mathrm{~mm}^{2}$. The impact mass was $4.98 \mathrm{~kg}$ and the drop height $64 \mathrm{~cm}$ to achieve an impact energy of $30.8 \mathrm{~J}$. A hemispherical-shaped steel tup of $12.7 \mathrm{~mm}$ in diameter was used as an impactor. The tup was instrumented with an accelerometer to measure the impact load, the tup displacement and the velocity.

The compressive strength after impact was determined according to ASTM standard D7137. Tests were carried out using an electromechanical universal testing machine (Instron 3384). The fixture incorporated adjustable side plates to accommodate the thickness variations and to prevent specimen buckling. The specimen was simply supported at the four edges, and the compression load was applied directly to the top fixture plate by a platen installed in the cross-head of the testing machine. The load was continuously measured during the test with a $150 \mathrm{kN}$ load cell. The instrumentation of the specimens included four back-to-back strain gages located at $25 \mathrm{~mm}$ from each edge to detect evidence of specimen bending during the test. The compressive strength after impact was determined from the maximum load and the initial cross section.

\subsection{X-ray computed microtomography}

The distribution of voids in the consolidated laminates was studied by XCT (Nanotom 160NF, Phoenix). The tomograms were collected at $50 \mathrm{kV}$ and $350 \mu \mathrm{A}$ using a Mo target. The resolution of the tomograms was set to $9 \mu \mathrm{m} /$ voxel. One prismatic sample for each curing cycle of $20 \times 20 \mathrm{~mm}^{2}$ and $4.6 \mathrm{~mm}$ in thickness was extracted for the tomographic inspections. Typical acquisition time for each tomogram was $5 \mathrm{~h}$. The tomograms were reconstructed using a commercial software (datos $\mid \mathrm{X}$ from Phoenix |X-rays) which algorithm is based on the filtered backprojection procedure for Feldkamp cone beam geometry.

Evaluation of the reconstructed volume focused on the void population. For that purpose, voids were extracted by identifying the voxels belonging either to a void or to the bulk composite material based on their gray level. The threshold for void segmentation was based on the local variance method from Sauvola applied to each slice, adapting the threshold according to the mean and standard deviation of the peak of the histogram (more details can be found in [13]). A cross section of the volume before and after the threshold procedure is shown in Fig. 1. A void was defined as the configuration of voxels sharing a common face, edge, or corner. Only voids larger than $2 \times 2 \times 2$ or more connected voxels were considered, as smaller voids may be artifacts from noise. The quantitative values of the volume fraction, distribution and geometry of voids were obtained from the binarized volumes. The specimen with the lowest porosity contained 13.736 pores of different sizes. The size of the representative volume for tomography was chosen as a compromise among several factors: the resolution (the smaller the sample the higher the achievable resolution) for the detection of the smallest pores, the detection of a sufficiently large number of pores for statistical relevance and a sufficiently large specimen volume to contain the largest pores in the samples. In addition, the damage introduced by low-velocity impact in the laminates was studied by means of XCT, as in previous studies [14,15].

\section{Curing cycles}

Three different thermal histories were used to cure the prepregs (Fig. 2a). Cycle $\mathrm{C}-1$ was recommended by the prepreg manufacturer for autoclave processing although with a faster heating rate. In this cycle, the laminate is heated at a constant rate up to $180^{\circ} \mathrm{C}$ and maintained at this temperature for $20 \mathrm{~min}$. Cycles C-2 and C-3 were designed on the basis of previous studies of rheological behavior of the AS4/8552 prepreg under isothermal conditions [4]. They were intended to enlarge the processing window by maintaining the material for a longer time at a low viscosity level, so void diffusion and transport was easier. The laminate was heated at a constant rate up to $130^{\circ} \mathrm{C}$ in cycle C-2 and the prepreg was held at this temperature for $10 \mathrm{~min}$ before heating up to $180^{\circ} \mathrm{C}$ was resumed. Cycle $\mathrm{C}-3$ presented a temperature ramp until $180^{\circ} \mathrm{C}$ (as in cycle $\mathrm{C}-1$ ) but the temperature was immediately reduced to $130^{\circ} \mathrm{C}$ after reaching the peak, held at this temperature for $10 \mathrm{~min}$ and finally increased up to $180^{\circ} \mathrm{C}$ again. In all cycles, once the laminate reached $180^{\circ} \mathrm{C}$ for the last time, it was held at this temperature during $120 \mathrm{~min}$ and cooled afterwards to ambient temperate at a constant rate of $10^{\circ} \mathrm{C} / \mathrm{min}$.

The prepreg viscosity profiles corresponding to each cure cycle are plotted in Fig. $2 \mathrm{~b}$. The gel point for each cycle was determined from the prepreg viscosity profile according to the Winter and Chambon criterion [12] and is marked with a vertical arrow in
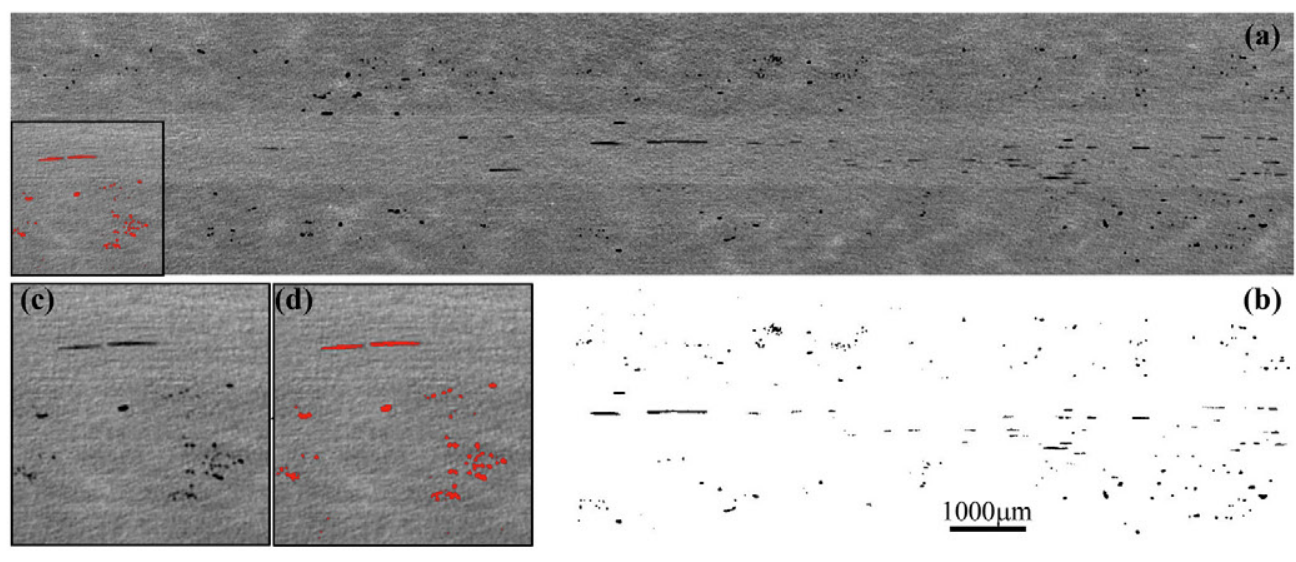

Fig. 1. Tomographic cross section before (a) and after (b) the threshold procedure. (c) and (d) are magnified pictures of the region depicted in (a). The section was obtained from the panel with $\left[45_{3} / \mathrm{O}_{3} /-45_{3} / 90_{3}\right]_{s}$ lay-up manufactured following the curing cycle $\mathrm{C}-2$. 

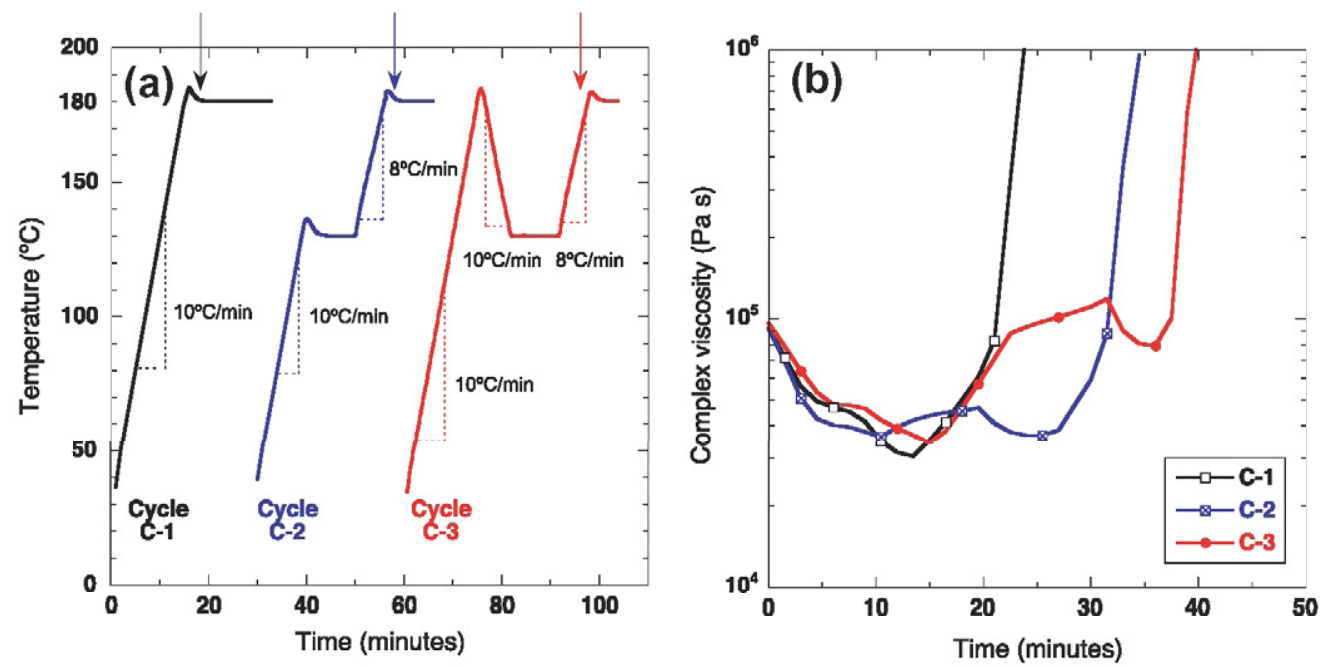

Fig. 2. (a) Profile of the three temperature cycles used to cure the quasi-isotropic panels. Maximum cure temperature was $180^{\circ} \mathrm{C}$ and heating and cooling ramps were carried out at $8^{\circ} \mathrm{C} / \mathrm{min}$ or $10^{\circ} \mathrm{C} / \mathrm{min}$, as indicated. Vertical arrows indicate the gel point corresponding to each cure cycle. The origin of the curves corresponding to cure cycles $\mathrm{C}-2$ and C-3 have been displaced by 30 and 60 min, respectively, for the sake of clarity. (b) Evolution of the complex viscosity $\eta^{*}$ for each cure cycle.

Table 1

Gel time, $t_{\text {gel }}$, degree of cure, $\alpha$, and glass transition temperature, $T_{g}$, of AS4/8552 composite panels manufactured with different curing cycles.

\begin{tabular}{llll}
\hline Cure cycle & $t_{\text {gee }}(\mathrm{min})$ & $\alpha$ & $T_{g}\left({ }^{\circ} \mathrm{C}\right)$ \\
\hline $\mathrm{C}-1$ & 17 & $0.891 \pm 0.013$ & $207.6 \pm 1.6$ \\
$\mathrm{C}-2$ & 28 & $0.895 \pm 0.022$ & $210.4 \pm 0.32$ \\
$\mathrm{C}-3$ & 36 & $0.896 \pm 0.018$ & $210.6 \pm 0.71$ \\
\hline
\end{tabular}

Fig. 2a. Gelification did not take place in any cycle before the maximum temperature of $180^{\circ} \mathrm{C}$ was attained for the second time, which guarantees enough time for resin flow and void evacuation. Cycle $\mathrm{C}-1$ begins with a marked reduction in viscosity from an initial value of $10^{5} \mathrm{~Pa}$ s to $3.0 \times 10^{4} \mathrm{~Pa}$ after $15 \mathrm{~min}$ because the temperature increase dominated over the curing reactions. Beyond this point, however, the trend was reversed and cross-linking of the epoxy led to a sharp increase in the viscosity, leading to a short processing window. The initial viscosity profile of cycle $\mathrm{C}-3$ was identical to cycle $\mathrm{C}-1$, as the thermal histories coincided. Reducing the cure temperature immediately after the peak was able to enlarge the processing window and the complex viscosity after $30 \mathrm{~min}$ was equivalent to the initial viscosity. Better results were obtained with cycle $\mathrm{C}-2$, which presented a wide processing window (from minute 10 to 30 ) with low viscosity $\left(\approx 4.0 \times 10^{4} \mathrm{~Pa} \mathrm{~s}\right.$ ).

The final degree of cure, $\alpha$, and the glass transition temperature, $T_{g}$, corresponding to each curing cycle, were determined by DSC. Two tests were carried out for each curing cycle and the average values (together with the standard deviation of $T_{g}$ and the experimental error in $\alpha$ ) are shown in Table 1 . They were very similar for all cycles, indicating that the mechanical properties of the resin were equivalent. The fiber volume fraction, obtained from the thermogravimetric analysis, was $60 \%$ in all cases.

\section{Results and discussion}

\subsection{Void volume fraction}

$\mathrm{XCT}$ provides an accurate assessment of the void volume fraction, shape and spatial distribution within the panels, much more precise than the information obtained from other non-destructive techniques or from measurements carried out on cross-sections of

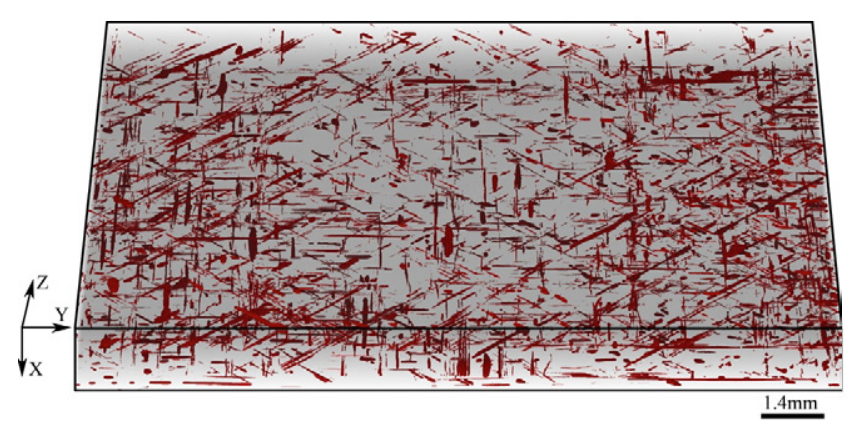

Fig. 3. X-ray microtomography of void spatial distribution in the quasi-isotropic $\left[45_{3} / 0_{3} /-45_{3} / 90_{3}\right]_{s}$ panel manufactured following cure cycle C-3.

Table 2

Volume fraction of voids, $V_{f}$, (expressed in \%) as a function of the cure cycle and laminate lay-up for AS4/8552 composite panels.

\begin{tabular}{llll}
\hline Curing cycle & {$[0]_{10}$} & {$\left[45_{3} / 0_{3} /-45_{3} / 90_{3}\right]_{s}$} & {$[45 / 0 /-45 / 90]_{3 s}$} \\
\hline $\mathrm{C}-1$ & 2.9 & 1.30 & 1.78 \\
$\mathrm{C}-2$ & 0.4 & 0.24 & 0.12 \\
$\mathrm{C}-3$ & 1.1 & 0.26 & 0.60 \\
\hline
\end{tabular}

the panels using optical or electron microscopy. The general distribution of the porosity within the quasi-isotropic $\left[45_{3} / 0_{3} /-45_{3} /\right.$ $\left.90_{3}\right]_{s}$ panel manufactured following the curing cycle $C-3$ is shown in Fig. 3. Matrix and fibers were set to semi-transparency to reveal the voids, which were elongated and oriented along the fiber direction (either $0^{\circ}, 90^{\circ},-45^{\circ}$ or $45^{\circ}$ ) in each ply. The void volume fraction of each quasi-isotropic laminate cured following the cycles $\mathrm{C}$ $1, \mathrm{C}-2$ and $\mathrm{C}-3$ is shown in Table 2, together with data obtained for uniaxial laminates $[0]_{10}$ of the same material cured with the same temperature cycles. The relationship between the processing window and the porosity is evident from this data. The minimum porosity was attained with cycle $\mathrm{C}-2$, while cycle $\mathrm{C}-1$ led to the highest porosity because there was not enough time to evacuate the voids from the laminate and they did not collapse in the absence of high hydrostatic pressure. Cycle C-3 also led to low porosities but the volume fraction of voids was consistently higher than that obtained with cycle $\mathrm{C}-2$. 


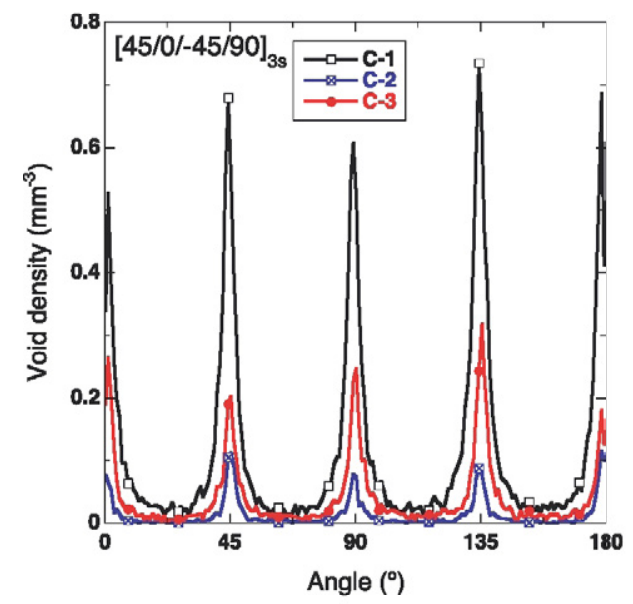

Fig. 4. Void density (expressed as the number of voids per $\mathrm{mm}^{3}$ ) as a function of the orientation of the major axis of the equivalent ellipsoid for the quasi-isotropic [45] $0 /-45 / 90]_{3 s}$ dispersed laminates processed with different cure cycles. Voids oriented at $135^{\circ}$ were parallel to the fibers in the $-45^{\circ}$ plies, while voids oriented at $0^{\circ}$ and $180^{\circ}$ were parallel to the fibers in the $0^{\circ}$ plies.

In order to assess the variation of the porosity throughout the panels, the porosity was measured by XCT in samples from different regions of the laminate cured following cycle C-2. It was found that the porosity remained practically constant (with a variation within $\pm 0.1 \%$ ) in the central region of the laminate and only increased when the distance to the border was below $25 \mathrm{~mm}$. Thus, the specimens for the evaluation of the mechanical properties were extracted from the central region of the laminate.

\subsection{Void orientation and spatial distribution}

In order to obtain quantitative information about the void orientation, shape and spatial distribution, each void was fitted to an equivalent cylinder of elliptical section whose volume, centroid and moments of inertia were equal to those of the void. Voids were elongated and the major axis was oriented in each ply parallel to the fibers. Thus, voids were mainly oriented in the $0^{\circ}, 90^{\circ},-45^{\circ}$ or $45^{\circ}$ directions, as shown in Fig. 4 , which presents the void density (number of voids per $\mathrm{mm}^{3}$ ) as a function of the orientation of the major axis of the equivalent ellipsoid. Void orientation was independent of the cure cycle and of the lay-up, although only the results corresponding to the dispersed laminate, [45/0/-45/ $90]_{3 s}$, are shown in Fig. 4 for the sake of brevity.

Fig. 5a shows the distribution of porosity along the width ( $Y$ axis) in a single cluster of plies with fibers oriented along the $Z$ axis in the $\left[45_{3} / 0_{3} /-45_{3} / 90_{3}\right]_{s}$ panel manufactured with cycle $C-3$. It should be noted that the surrounding air at the edge of the specimen is segmented as voids since both features have the same absorption coefficient. This could lead to an improper evaluation of the void volume fraction close to the specimen edge. Therefore the sample volume was cropped down to $16 \mathrm{~mm}$ in the $Y$ direction to avoid this effect. Geometry of the individual voids was, however, evaluated in the $20 \times 20 \mathrm{~mm}^{2}$ specimen and all voids not touching the surface were fitted to a cylinder and included in the statistical analysis. The void volume fraction corresponding to each point of Fig. 5a (and Fig. 6) was obtained by averaging the void volume fraction in slices of $45 \mu \mathrm{m}$ ( 5 voxels) in thickness perpendicular to the $Y$ axis (or to the $X$ axis in Fig. 6).

These results show that the porosity was inhomogeneously distributed along the width of each ply, following an approximately periodic pattern with peaks of high porosity (in the range 3-10\%) separated by valleys with zero porosity. More information can be obtained from Fig. 5b, which shows the X-ray absorption of the same cluster of plies along the fiber axis. This image was obtained by averaging the gray values of parallel slices along the $Z$ axis and takes advantage of the concentration of voids in channels parallel to the fibers and of the differences in density (and, thus, in X-ray absorption) between voids, resin and fibers. Black zones stand for low density sections which contain very high porosity. White zones represent high fiber density sections while gray zones are either low fiber density or resin-rich regions. Porosity was mainly concentrated within resin-rich tubular cells, and this spatial distribution was also found in unidirectional laminates [4] as a result of a process of inhomogeneous consolidation during curing. Upon the application of pressure, most of the load is transferred through a continuous skeleton of fiber-rich regions. The higher pressure in these regions leads to the migration of resin as well as voids into

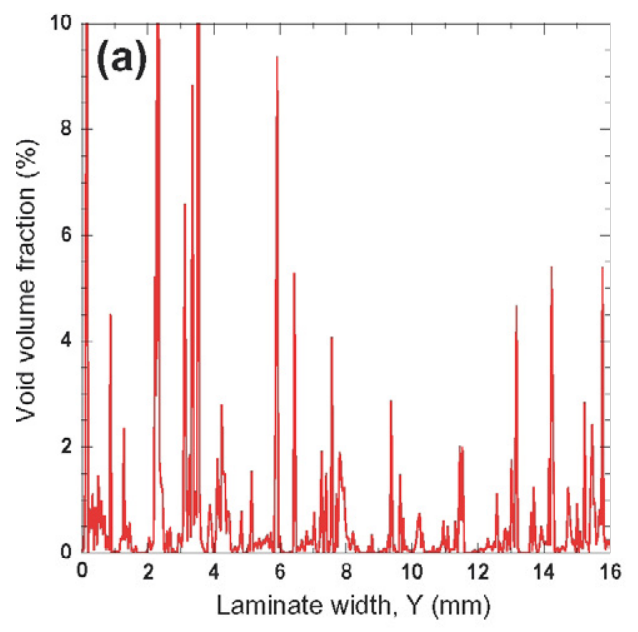

(b)

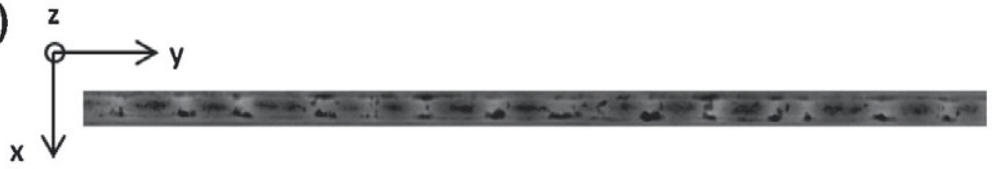

Fig. 5. (a) Distribution of porosity along the width (Y axis) in a single cluster of plies with fibers oriented along the $\mathrm{Z}$ axis in the $\left[45_{3} / 0_{3} /-45_{3} / 90_{3}\right]_{s}$ panel manufactured with the cure cycle C-3 (b) X-ray absorption along the fiber ( $Z$ axis) of the same single cluster of plies. 

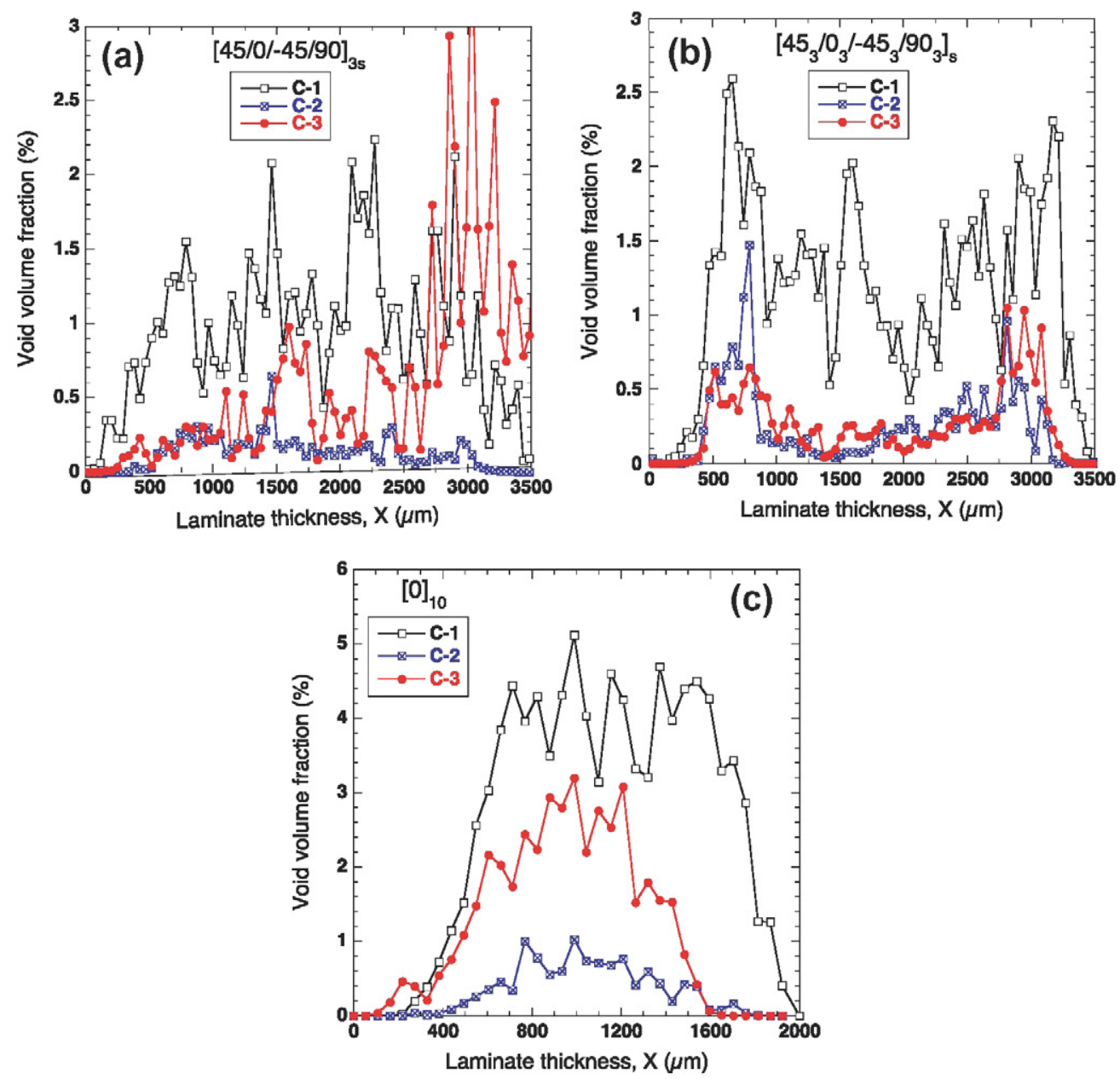

Fig. 6. Porosity distribution through the thickness (Xaxis) of the laminates subjected to different curing cycles. (a) $[45 / 0 /-45 / 90]_{3 s}$ dispersed laminate. (b) $\left[45_{3} / 0_{3} /-45_{3} / 90_{3}\right]_{s}$ clustered laminate. (c) $[0]_{90}$ unidirectional laminate (from [4]).

the cells formed by the skeleton. The pressure in these regions is lower, facilitating the nucleation and/or growth of voids. Thus resin flow in each ply was anisotropic and mainly occurred along the fiber direction, in agreement with the higher permeability factor in this direction. The dominant resin flow along the fibers led to the formation of a channel-type structure (also reported in previous studies $([7,16]))$ and facilitated the transport and coalescence of voids along the fibers.

The spatial distribution of the voids through the laminate thickness ( $X$ axis) is shown in Fig. $6 \mathrm{a}$ and $\mathrm{b}$ for the dispersed ([45/0/-45/ $90]_{3 s}$ ) and clustered $\left(\left[45_{3} / 0_{3} /-45_{3} / 90_{3}\right]_{s}\right)$ laminates, respectively. The porosity was zero close to the laminate surfaces in all cases because voids in these areas could easily migrate outside of the panel. Porosity increased with the distance to the surfaces and two different distributions were found depending on the laminate sequence. In the case of the dispersed laminate (Fig. 6a), the porosity was - on average - constant through the thickness while it reached a maximum value at approximately $1 \mathrm{~mm}$ from the surfaces ${ }^{1}$ in the clustered laminates and then decreased rapidly to a plateau in the central region (Fig. 6b). Overall. the porosity profile was similar in dispersed and clustered laminates but it differed from the one measured in unidirectional $[0]_{10}$ panels [4], which is shown in Fig. $6 \mathrm{c}$. The porosity increased from the surfaces towards the interior of the panel in this case and the void volume fraction in the center of the

\footnotetext{
${ }^{1}$ It should be noted that the average laminate thickness was $\approx 4.6 \mathrm{~mm}$ but $\approx 0.5 \mathrm{~mm}$ in each surface were not included in the tomographic analysis.
}

panel for unidirectional laminates was much higher than that found in multiaxial panels subjected to the same curing cycle. These results seem to indicate that interplies between lamina with different fiber orientation also acted as pathways for void migration during consolidation. Thus, the porosity of multi axial laminates was lower than that of unidirectional panels for identical curing conditions (Table 2). Panel examination after processing revealed resin bleeding on all faces in the multidirectional laminates while only on the faces perpendicular to the fiber direction in the unidirectional panels.

\subsection{Void shape}

In addition to the void volume, the void shape was characterized by the elongation factor $e=2 b_{1} /\left(b_{2}+b_{3}\right)$ and the flatness ratio $f=b_{2} / b_{3}$ where $b_{1}, b_{2}$ and $b_{3}$ stand for the maximum, medium and minimum principal semi-axes of the equivalent ellipsoid, respectively. The minor axis was perpendicular to the laminate. The average length of the major and minor axes of the equivalent ellipsoid are plotted as a function of the individual void volume in Fig. 7a and b, respectively, for the dispersed ([45/0/-45/90 $\left.]_{3 s}\right)$ laminate and in Fig. 8a and b for the clustered $\left(\left[45_{3} / 0_{3} /-45_{3}\right]\right.$ $90_{3} l_{s}$ ) panels. A bin size of $14.580 \mu \mathrm{m}^{3}$ (20 voxels) was selected for individual void volume and used in the abscissa axis of Figs. 7-9. The results for the medium axis $\left(b_{2}\right)$ followed the same trends and are not included for sake of brevity. These data show that the voids were elongated and that the void length increased rapidly with the void volume, indicating that the voids grew along 

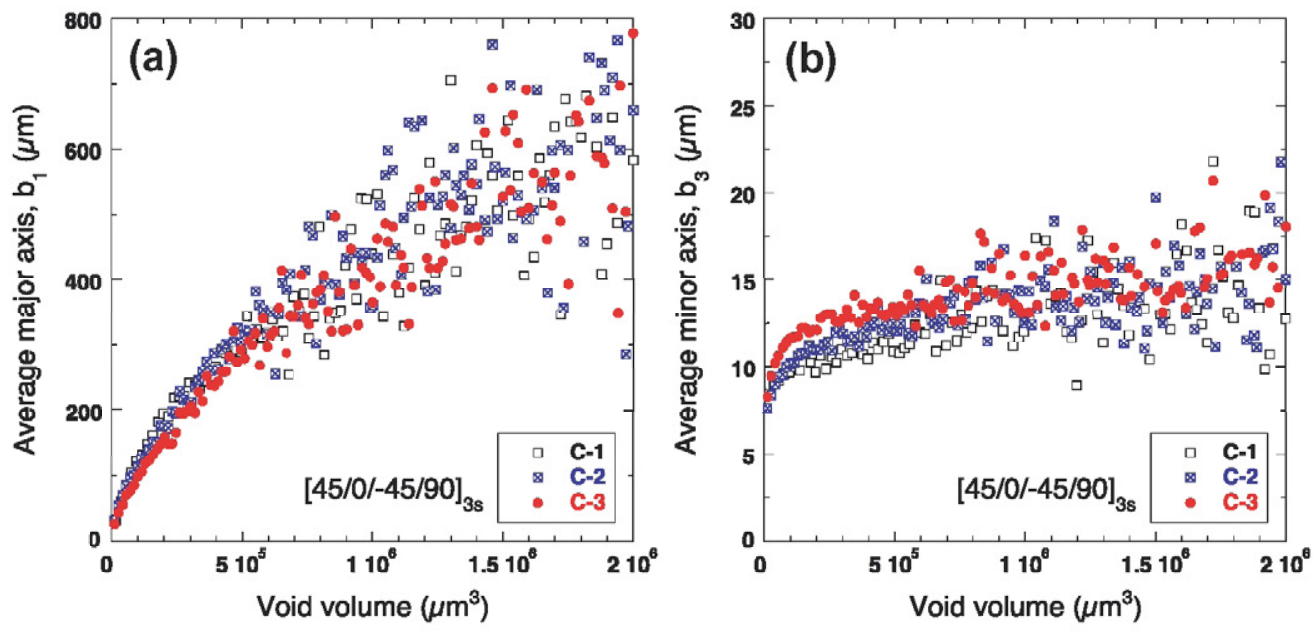

Fig. 7. Average length of the major axis of the equivalent ellipsoid, $b_{1}$, of the voids in the $[45 / 0 /-45 / 90]_{3 s}$ dispersed laminate. (b) Idem for the minor axis of the equivalent ellipsoid, $b_{3}$. Results are presented for panels cured using different cycles.
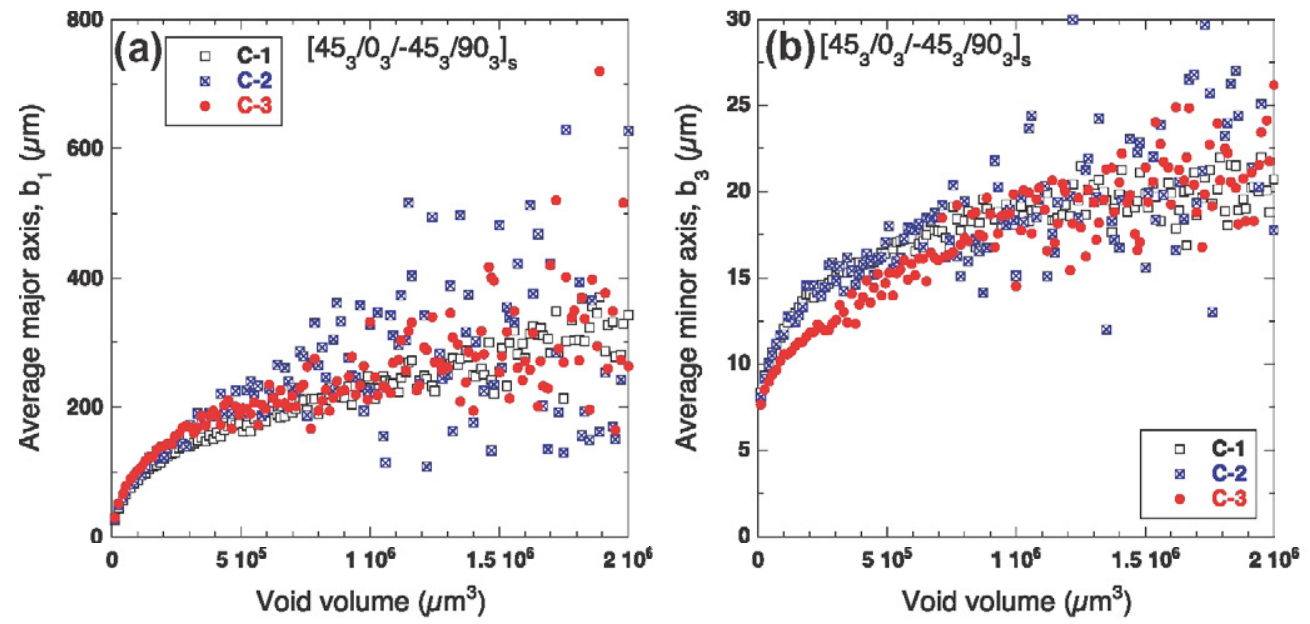

Fig. 8. Average length of the major axis of the equivalent ellipsoid, $b_{1}$, of the voids in the $\left[45_{3} / 0_{3} /-45_{3} / 90_{3}\right]_{s}$ clustered laminate. (b) Idem for the minor axis of the equivalent ellipsoid, $b_{3}$. Results are presented for panels cured using different cycles.

the fiber direction in each ply. Although the cure cycle influenced the void volume fraction (Table 2), it did not change the morphology of the voids and the length of the axes of the equivalent ellipsoid were independent of the cure cycle.

The comparison of Figs. 7 and 8 also indicated that the void morphology depended on the laminate lay-up. This is more evident in Fig. 9a and b which show the evolution of the average elongation factor, $e$, and of the flatness ratio, $f$, as function of the void volume for the clustered, dispersed and unidirectional laminates cured following cycle $\mathrm{C}-3$. Similar results were also found for curing cycles $\mathrm{C}-1$ and $\mathrm{C}-2$. It is worth noting that the elongation factor increased with the degree of clustering and this indicates that void migration along the fiber direction was favored by clustered plies of the same orientation, leading to more elongated pores. The flatness ratio, depicted in Fig. 9b, was equivalent in the unidirectional and clustered laminates and slightly higher in the dispersed laminate. These differences were not significant and indicate that the shape of the pore section was mainly controlled by the consolidation pressure.

\subsection{Compressive strength}

It is well established that porosity leads to a marked reduction in the laminate mechanical properties $[17,18]$, particularly those dominated by the matrix such as the interlaminar shear strength and the transverse tensile strength $[19,20]$. However, it was not always possible to distinguish the effect of porosity from other factors (such as ply clustering) because the porosity information was always limited. The detailed information of the pore volume fraction and spatial distribution obtained by XCT overcomes these limitations and allows a detailed study of the influence of porosity on the compressive strength.

The average elastic modulus $\left(E_{c}\right)$ and compressive strength $\left(X_{C}\right)$ obtained from 5 tests (together with the corresponding standard deviations) are plotted in Fig. 10a and b, respectively. The influence of the porosity on the elastic modulus was very small, as it could be expected from the low porosity levels in the laminates. Similarly, the modulus of the laminates with a dispersed lay-up was marginally higher but neither the porosity nor the lay-up configuration were relevant. This was not the case in the compressive strength, in which both factors did influence the mechanical performance. The compressive strength of the laminates processed following cycle $\mathrm{C}-1$ (with a porosity in the range $1.3-1.8 \%$ ) was smaller (by $\approx 50 \mathrm{MPa}$ in the clustered lay-up and by $100-150 \mathrm{MPa}$ in the dispersed laminate) than that of the plates processed following cycles $\mathrm{C}-2$ and $\mathrm{C}-3$, whose porosity was below $0.6 \%$. These results point out the importance of designing an optimum cure cycle as 

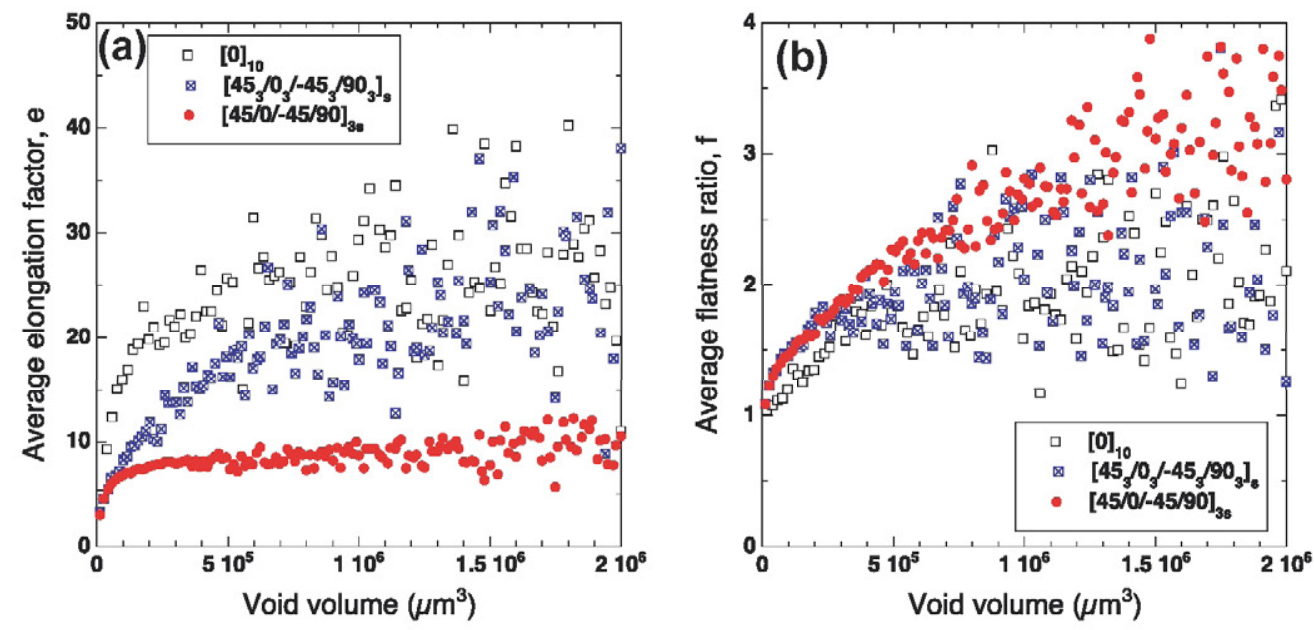

Fig. 9. (a) Average elongation factor, $e=2 b_{1} /\left(b_{2}+b_{3}\right)$, as a function of the void volume fraction for laminates with different lay-up cured using cycle $C$-3. (b) Idem for the average flatness ratio, $f=b_{2} / b_{3}$. Results for the unidirectional laminate were obtained from [4].
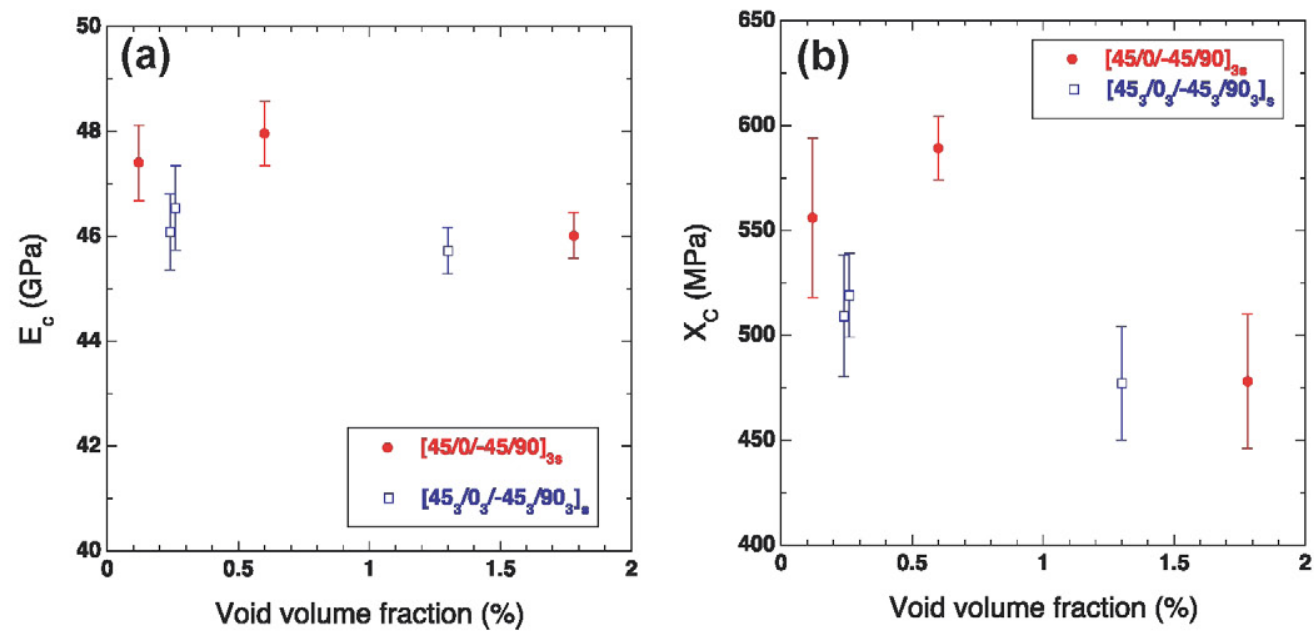

Fig. 10. (a) Elastic modulus, $E_{c}$ as a function of the void volume fraction. (b) Compressive strength, $X_{C}$, as a function of the void volume fraction.

limited porosity levels (below $2 \%$ ) led to a noticeable reduction in the compressive strength and this effect will be amplified for higher void volume fractions.

It is also worth noting that dispersed laminates presented higher compressive strength than clustered ones for equivalent values of the void volume fraction. Fracture occurred catastrophically in both materials and optical micrographs of the broken specimens showed that it was accompanied by delamination between plies with different fiber orientation (Fig. 11). Thus, delamination was concentrated in a few planes in the clustered laminates (Fig. 11a). The effect of ply clustering on the compressive strength of quasi-isotropic laminates was studied by Lee and Soutis [21]. Laminates with dispersed $\left([45 / 90 /-45 / 0]_{n s}\right)$ or clustered $\left(\left[45_{n} / 90_{n} /-45_{n} / 0_{n}\right]\right)$ lay up and different thickness $(n=2,4,8)$ were tested in compression and no differences in the compressive strength were found in the case of $n=2$ and 4 . In the case of the thicker laminates $(n=8)$, clustered laminates were significantly weaker but this behavior was attributed to pre-existing matrix cracking damage induced during machining in the thick plies. Our results do show an effect of ply-clustering for equivalent void content and it should be noted that this latter variable was not controlled in [21]. The differences in the compressive strength between clustered and dispersed laminates can be rationalized in terms of the critical stress necessary to trigger microbuckling in
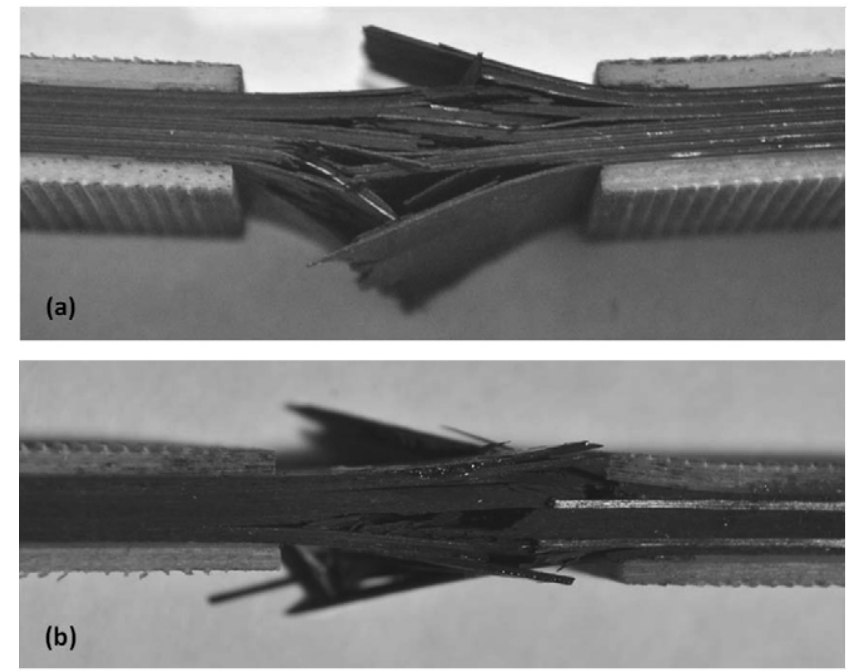

Fig. 11. Failure mode in compression of laminates cured with cycle $\mathrm{C}-2$. (a) $\left[45_{3} / 0_{3} /-45_{3} / 90_{3}\right]_{s}$ clustered laminate. (b) $[45 / 0 /-45 / 90]_{3 s}$ dispersed laminate.

the $0^{\circ}$ plies. Assuming that the fiber orientation was independent of the ply thickness, this stress depends on the confinement 

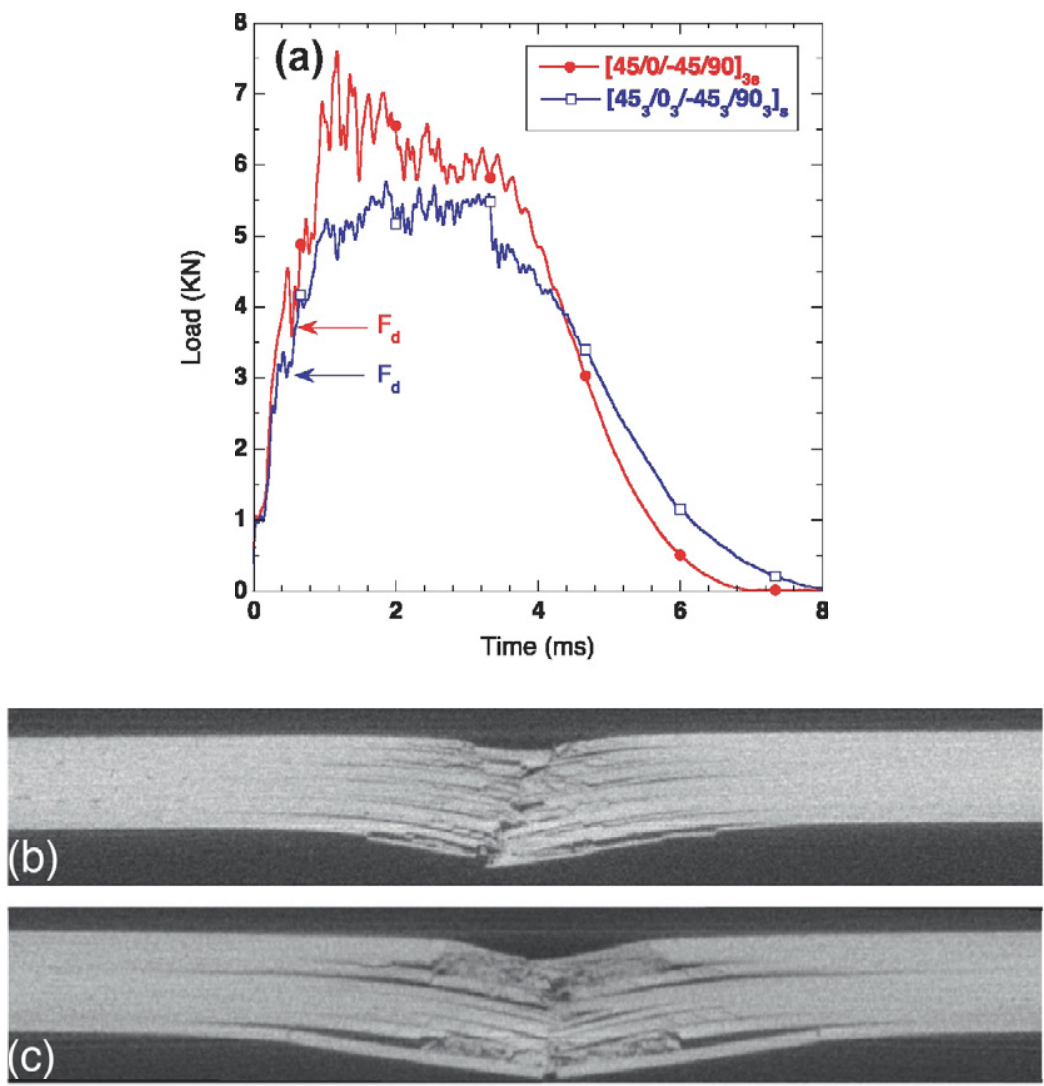

Fig. 12. (a) Load vs. time curves of the low-velocity impact tests carried out in dispersed and clustered laminates cured with cycle C-2 (b) Tomogram of the $[45 / 0 /-45 / 90]_{3 s}$ dispersed laminate after low-velocity impact. (c) Tomogram of the $\left[45_{3} / 0_{3} /-45_{3} / 90_{3}\right]_{s}$ clustered laminate after low-velocity impact.

provided by the contiguous plies with different fiber orientation and drops rapidly once interply delimitation occurs. It is well known that interply delamination occurs at lower stresses in clustered laminates because they are prone to matrix cracking and there is more elastic energy stored in the ply to promote delamination [22]. Thus, early interply delamination is expected in clustered materials and will trigger catastrophic failure at lower stresses.

\subsection{Compressive strength after impact}

Representative load vs. time curves of the low-velocity impact tests are plotted in Fig. 12a for the dispersed and clustered laminates cured with cycle $\mathrm{C}-2$. Cure cycle (and, thus porosity) did not influence these curves and the corresponding curves for cure cycles $\mathrm{C}-1$ and $\mathrm{C}-3$ are not repeated. Laminates with different lay-up showed, however, a different response, following the trends outlined in [23]. In particular, dispersed laminates showed higher delamination threshold, $F_{d}$, (marked with arrows in Fig. 12a) as well as higher peak loads. In addition, the changes in stiffness during impact become more progressive and smoother in clustered laminates and the response in time was longer. Finally, the total energy absorbed during impact was independent of the ply thickness. These behaviors can be correlated to the failure micromechanisms depicted in Fig. 12b and c for the dispersed and clustered laminates, respectively, cured following cycle $\mathrm{C}-2$. The tomograms of the cross-section below the impactor show that the main damage mechanisms were interply delamination as well as intraply failure. Delaminations occurred between plies with different orientation. They were evenly distributed through the thickness and had similar length in the dispersed laminate (Fig. 12b), while fewer though much longer delaminations were found in the clustered material (Fig. 12c) and the residual crack openings were wider.

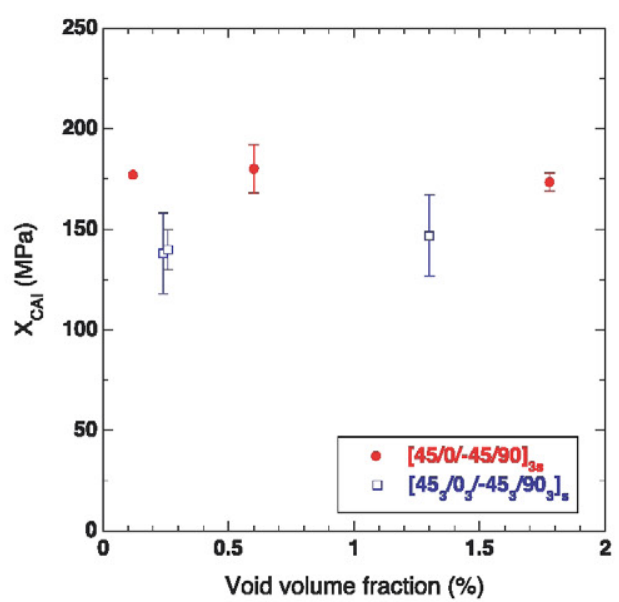

Fig. 13. Compressive strength after impact, $X_{\mathrm{CAl}}$, as a function of the void volume fraction.

Moreover, intraply damage in the form of crushing below the impact was higher in the clustered material. These observations are in agreement with the curves in Fig. 12a. The critical load for delamination was smaller in the clustered material but delaminations were localized in a limited number of interplies and grew longer, leading to a high delaminated area. As a result, the stiffness of the clustered material decreased rapidly after the development of interply decohesion leading to a reduction in the peak load and to a longer time response.

The average compressive strength after impact obtained from 3 tests (together with the corresponding standard deviations) is 
plotted in Fig. 13 for all the laminates. All specimens failed catastrophically at the maximum load. These results show that damage induced during impact overcame the effect of porosity on the compressive strength and that the residual strength of clustered laminates was consistently lower than that of plates with a dispersed lay-up. These data are in agreement with the damage patterns presented in Fig. 12b and c, which showed longer and wider delaminations in the clustered laminate. They should facilitate micro buckling of the $0^{\circ}$ plies under compression.

\section{Conclusions}

Quasi-isotropic carbon fiber-epoxy laminates were processed by compression molding to assess the effect of the curing cycle and of the laminate lay-up (either dispersed or clustered) on the void volume fraction, spatial distribution and shape and on the compressive strength before and after impact. It was found that optimized quasi-isotropic laminates with very low porosity $(\leqslant 0.5 \%)$ can be manufactured out-of-autoclave under low pressure $(0.2 \mathrm{MPa})$ by a careful design of the temperature cycle, leading to a wide processing window in which the resin has low viscosity. Void migration during consolidation took place along the interplies between lamina with different fiber orientation. Within the plies, porosity was localized in channels formed by elongated voids parallel to the fibers, indicating that void migration occurred parallel to the fibers in the direction of resin flow.

The compressive strength of the laminates decreased as the void volume fraction increased. Dispersed laminates presented higher compressive strength than clustered ones for equivalent levels of porosity. This result was attributed to the early onset of delamination upon loading in the laminate with thicker plies. Similarly, lower peak loads and delamination thresholds as well as more extended delaminations were found in clustered laminates subjected to low-velocity impact. In addition, they showed lower compression after impact strength. Porosity did not influence either the low-velocity impact behavior or the compression after impact as defects introduced during impact overcame the influence of porosity on the failure mechanisms.

\section{Acknowledgements}

This investigation was supported by the EU 6thFP through the project DEFCOM (EraNet MATERA) and by Spanish Ministry of Economy and Competitiveness through the project MUDATCOM (MAT2012-37552). FS also acknowledges the scholarship from the AMAROUT programme (Marie Curie actions, EU 7thFP).

\section{References}

[1] Nightingale C, Day R. Flexural and interlaminar shear strength properties of carbon fibre/epoxy composites cured thermally and with microwave radiation. Composites: Part A 2002;33:1021-30.

[2] Davies LW, Day RJ, Bond D, Nesbitt A, Ellis J, Gardon E. Effect of cure cycle heat transfer rates on the physical and mechanical properties of an epoxy matrix composite. Compos Sci Technol 2007;67:1892-9.

[3] Grunenfelder LK, Nutt SR. Void formation in composite prepregs $\bar{D}$ effect of dissolved moisture. Compos Sci Technol 2010;70:2304-9.

[4] Hernández S, Sket F, Molina-Aldareguía JM, González C, LLorca J. Effect of curing cycle on void distribution and interlaminar shear strength in polymermatrix composites. Compos Sci Technol 2011;71:1331-41.

[5] Agius SL, Magniez KJC, Fox BL. Cure behaviour and void development within rapidly cured out-of-autoclave composites. Composites: Part B 2013;47:230-7.

[6] Lee WI, Loos AC, Springer GS. Heat reaction, degree of cure and viscosity of Hercules 3501-6 resin. J Compos Mater 1982;16:510-20.

[7] Loos AC, Springer GS. Curing of epoxy matrix composites. J Compos Mater $1983 ; 17: 135-69$

[8] Terzaghi K. Theoretical soil mechanics. John Wiley \& Sons; 1943.

[9] Kardos JL, Dudukovic MP, Dave R. Void growth and resin transport during processing of thermosetting $\bar{D}$ matrix composite. Adv Polym Sci 1986;80:101-23.

[10] Ledru Y, Bernhart G, Piquet R, Schmidt F, Michel L. Coupled visco-mechanical and difussion void growth modeling during composite curing. Compos Sc Technol 2010;70:2139-45.

[11] Hexcel, Hexply 8552 epoxy matrix product data; 2010. <www.hexcel.com>.

[12] Winter HH, Chambon F. Analysis of linear viscoelasticity of a crosslinking polymer at the gel point. J Rheol 1986;30:367-82.

[13] Yan F, Zhang H, Kube CR. A multistage adaptive thresholding method. Pattern Recog Lett 2005;26:1183-91.

[14] Enfedaque A, Molina-Aldareguía JM, Gálvez F, González C, LLorca J. Effect of glass-fiber hybridization on the behavior under impact of woven carbon fiber/ epoxy laminates. J Compos Mater 2010;44:3051-68.

[15] Seltzer R, González C, Muñoz R, LLorca J, Blanco-Varela T. X-ray microtomography analysis of the damage micromechanisms in 3D woven composites under low-velocity impact. Composites: Part A 2013;45:49-60.

[16] Tang J-M, Lee WI, Springer GS. Effects of cure pressure on resin flow, voids and mechanical properties. J Compos Mater 1987;21:421-40.

[17] Judd NCW, Wright WW. Voids and their effects on the mechanical properties of composites - an appraisal. SAMPE J 1978;14:10-4.

[18] Liu L, Zhang B, Wu Z, Wang D. Effect of cure pressure induced voids on the mechanical strength of carbon/epoxy laminates. J Mater Sci Technol 2005;21:87-91.

[19] Liu L, Zhang B-M, Wang D-F, Wu Z-J. Effects of cure cycles on void content and mechanical properties of composite laminates. Compos Struct 2006;73:303-9.

[20] Bowles K, Frimpong S. Voids effects on the interlaminar shear strength of unidirectional graphite-fiber-reinforced composites. J Compos Mater $1992 ; 26: 1487-509$.

[21] Lee J, Soutis C. A study of the compressive strength of thick carbon fibre-epoxy laminates. Compos Sci Technol 2007;67:2015-26.

[22] Wisnom MR, Hallet SR, Soutis C. Scaling effects in notched composites. J Compos Mater 2010;44:195-210.

[23] González EV, Maimí P, Camanho PP, Lopes CS, Blanco N. Effects of ply clustering in laminated composite plates under low-velocity impact loading. Compos Sci Technol 2011;71:805-17. 